\title{
Stereoselective Pharmacokinetics of BOF-4272 Racemate after Oral Administration to Rats and Dogs
}

\author{
Shinsaku NAITO* and Masuhiro NishimURA \\ Laboratory of Drug Metabolism Research, Naruto Research Institute, Otsuka Pharmaceutical Factory, Inc.; Naruto, \\ Tokushima 772-8601, Japan. Received September 25, 2001; accepted January 10, 2002
}

\begin{abstract}
The stereoselective pharmacokinetics of BOF-4272 enantiomers in rats and dogs was investigated by simultaneously measuring concentrations in arterial, portal, and venous plasma, the liver, and the kidney at $2 \mathrm{~h}$ after the oral administration of the racemic drug. The concentrations of BOF-4272 enantiomers were measured using high-performance liquid chromatography. The concentrations of the $S(-)$ enantiomer in arterial, portal, and venous plasma were higher than those of the $R(+)$ enantiomer in rats, but the opposite was found in dogs. In rats, absorption from the intestinal tract into the portal system was almost the same for the two enantiomers, whereas the hepatic uptake of the $R(+)$ enantiomer was greater than that of the $S(-)$ enantiomer. In dogs, absorption from the intestinal tract into the portal system was greater for the $R(+)$ enantiomer than for the $S(-)$ enantiomer, whereas hepatic uptake was comparable for the two enantiomers. The stereoselectivity of the renal uptake of BOF-4272 enantiomers had little effect on the stereoselectivity of enantiomers in the systemic circulation in both rats and dogs. The stereoselectivity in the systemic circulation of BOF-4272 enantiomers is therefore related to hepatic uptake in rats, and to absorption from the intestinal tract into the portal system in dogs.
\end{abstract} 4272

Key words stereoselectivity; xanthine oxidase inhibitor; sulphoxide-containing drug; oral administration; hepatic uptake; BOF-

BOF-4272, a derivative of pyrazolotriazine, is a new drug that has been developed for the treatment of hyperuricemia and ischemic reperfusion injury. ${ }^{1-4)}$ Since BOF-4272 contains an asymmetric sulphoxide group (Fig. 1), it exists as $R(+)$ and $S(-)$ enantiomers. Recently, an asymmetric synthesis of the $S(-)$ enantiomer of BOF-4272 was reported by Matsugi et al. ${ }^{5}$ BOF-4272 inhibits the de novo biosynthesis of uric acid by blocking the xanthine oxidase/xanthine dehydrogenase system, which catalyses the last step of purine catabolism. ${ }^{1,2)}$ The mechanism of the inhibitory action of BOF4272 was elucidated by an in vitro study using milk xanthine oxidase/xanthine dehydrogenase. ${ }^{6}$ ) BOF-4272 significantly decreases the concentration of free radicals generated by xanthine oxidase and consequently reduces cellular necrosis. ${ }^{7)}$ It was demonstrated in previous in situ studies that the hepatic elimination of BOF-4272 is quite high in rats, ${ }^{8-10)}$ and was also demonstrated in such a study that the hepatic extraction ratio of the $R(+)$ enantiomer of BOF-4272 was significantly greater than that of the $S(-)$ enantiomer in rats. ${ }^{11)}$

This paper describes the detailed stereoselectivity of BOF4272 enantiomers in rats and dogs after the oral administration of racemic BOF-4272.

\section{MATERIALS AND METHODS}

Materials The BOF-4272 used in this study was synthesised at Otsuka Pharmaceutical Factory, Inc. (Tokushima, Japan). Carboxymethyl cellulose sodium salt (CMC), ethyl acetate, diethyl ether, and perchloric acid (PCA) were purchased from Wako Pure Chemical Industries, Ltd. (Osaka, Japan). All other chemicals and reagents used were of analytical reagent grade.

Chromatography BOF-4272 concentrations were measured using high-performance liquid chromatography (HPLC) systems (CCP \& 8010 Series, Tosoh Co., Tokyo, Japan) with a stationary phase of LiChrospher $100 \mathrm{NH}_{2}$
( $250 \times 4.0$ mm I.D.; Merck, Darmstadt, Germany) on an extraction column and two CHIRALCELL ODs $(50 \times 4.6 \mathrm{~mm}$ I.D.; Daicel Chemical Industries, Ltd., Tokyo, Japan) on a chiral separation column. The concentrations of the BOF4272 enantiomers were determined using a fluorescence photometer (Tosoh Co.) with excitation and emission wavelengths of 319 and $400 \mathrm{~nm}$, respectively. The integrated data analyser used was a C-R4AX Chromatopac (Shimadzu Co., Kyoto, Japan). The flow rate and the column temperature were $0.6 \mathrm{ml} / \mathrm{min}$ and $43^{\circ} \mathrm{C}$, respectively. The mobile phase was a mixture of $n$-hexane, ethanol, and formic acid $(650: 350: 4 \mathrm{v} / \mathrm{v})$.

Animal Studies The rats used were male Wistar rats 7 weeks of age and weighing between 240 and $258 \mathrm{~g}(n=25)$ purchased from Charles River Japan (Kanagawa, Japan). The dogs used were male beagle dogs $8-9$ months of age and weighing between 9.17 and $10.1 \mathrm{~kg}(n=5)$ purchased from Covance Research Products, Inc. (Denver, PA, U.S.A.).

During the experiment, the rats and dogs were housed in individual metabolic cages at a temperature of $23 \pm 2{ }^{\circ} \mathrm{C}$ and a relative humidity of $55 \pm 10 \%$ on a $12-\mathrm{h}$ night/d cycle. The animals were allowed free access to food (rats, CRF-1-R; dogs, DS-5; Oriental Yeast Co., Ltd., Tokyo), except during
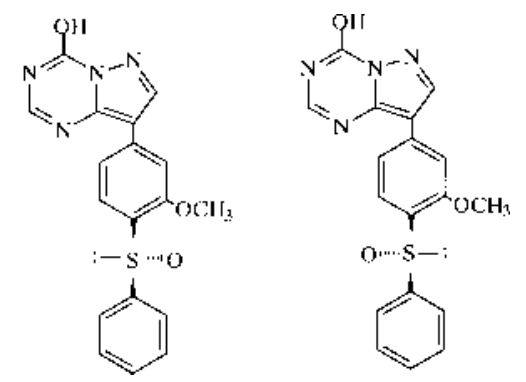

Fig. 1. Chemical Structure of BOF-4272

The structures of the $S(-)$ and $R(+)$ enantiomers of BOF-4272 are shown on the left and right, respectively. 
periods of fasting as described below, and to drinking water. Animals were fasted from $16 \mathrm{~h}$ before drug administration to the time of blood sampling. Racemic BOF-4272 was dissolved in $0.5 \% \mathrm{CMC}$ solution for oral administration to rats, and was placed in gelatine capsules for oral administration to dogs. It was administered orally to fasted animals at a dose of $25 \mathrm{mg} / \mathrm{kg}$. Rats were anaesthetised with diethyl ether, and dogs with pentobarbital $(50 \mathrm{mg} / \mathrm{kg}$, i.p.; Nembutal, Abbott Laboratories, North Chicago, IL, U.S.A.), and blood samples were obtained. In rats, blood samples were drawn from both the portal vein and the abdominal aorta into heparinized test tubes at $0.5,1,2,4$, and $8 \mathrm{~h}$ after oral administration. In both rats and dogs, blood samples were also drawn from three points (the portal vein, the inferior vena cava, and the abdominal aorta) into heparinized test tubes at $2 \mathrm{~h}$ after oral administration. All blood samples were immediately centrifuged to obtain plasma. The livers and kidneys of the rats and dogs were perfused with saline solution at $4{ }^{\circ} \mathrm{C}$, resected, and frozen on dry ice.

Preparation of HPLC Samples from Rat and Dog Plasma Each plasma sample $(0.5 \mathrm{ml})$ was mixed with ethyl acetate $(3 \mathrm{ml})$ and shaken. After centrifugation, $2 \mathrm{ml}$ of the ethyl acetate layer was transferred to another tube and dried under reduced pressure. The dried sample was dissolved in $200 \mu \mathrm{l}$ of the mobile phase for analysis of BOF-4272, shaken, and filtered through a membrane filter (pore size, $0.2 \mu \mathrm{m})$. A $50-\mu 1$ aliquot of the filtrate was injected onto the HPLC system for analysis of BOF-4272.

Preparation of HPLC Samples from Rat and Dog Liver and Kidney Each frozen liver sample (about $500 \mathrm{mg}$ ) was prepared by the successive addition of 5 volumes of PCA at $4{ }^{\circ} \mathrm{C}$ with shaking. After centrifugation, the supernatant was transferred to another tube, mixed with ethyl acetate $(4 \mathrm{ml})$, and shaken again. After a second centrifugation, $1 \mathrm{ml}$ of the ethyl acetate layer was transferred to another tube and dried under reduced pressure. The dried sample was dissolved in $200 \mu \mathrm{l}$ of the mobile phase for analysis of BOF-4272, shaken, and filtered through a membrane filter (pore size, $0.2 \mu \mathrm{m})$. A $50-\mu \mathrm{l}$ aliquot of the filtrate was injected onto the HPLC system for analysis of BOF-4272.

Data Analysis All experimental results for rats and dogs are given as the mean \pm S.D. of 10 and 5 animals, respectively. $\Delta \mathrm{Cp}$ is the difference between the portal plasma concentration and the arterial plasma concentration. $\mathrm{Kp}$ is the ratio of the liver or kidney concentration to the arterial plasma concentration. Statistical analysis was performed using the paired Student's $t$-test (two-tailed) with a significance level of $p<0.05$.

\section{RESULTS}

Figure 2 shows the plasma concentrations in the artery and the portal vein as well as the ratios of arterial to portal plasma concentrations (A/P ratios) of both enantiomers of BOF-4272 after the oral administration of racemic BOF4272 to rats. Panels $\mathrm{A}, \mathrm{B}$, and $\mathrm{C}$ show the arterial plasma concentrations, the portal plasma concentrations, and the $\mathrm{A} / \mathrm{P}$ ratios, respectively, of both enantiomers. The arterial plasma concentrations of the $S(-)$ enantiomer were significantly higher than those of the $R(+)$ enantiomer from $0.5 \mathrm{~h}$ to $4 \mathrm{~h}$ after oral administration (Fig. 2A). There were no significant
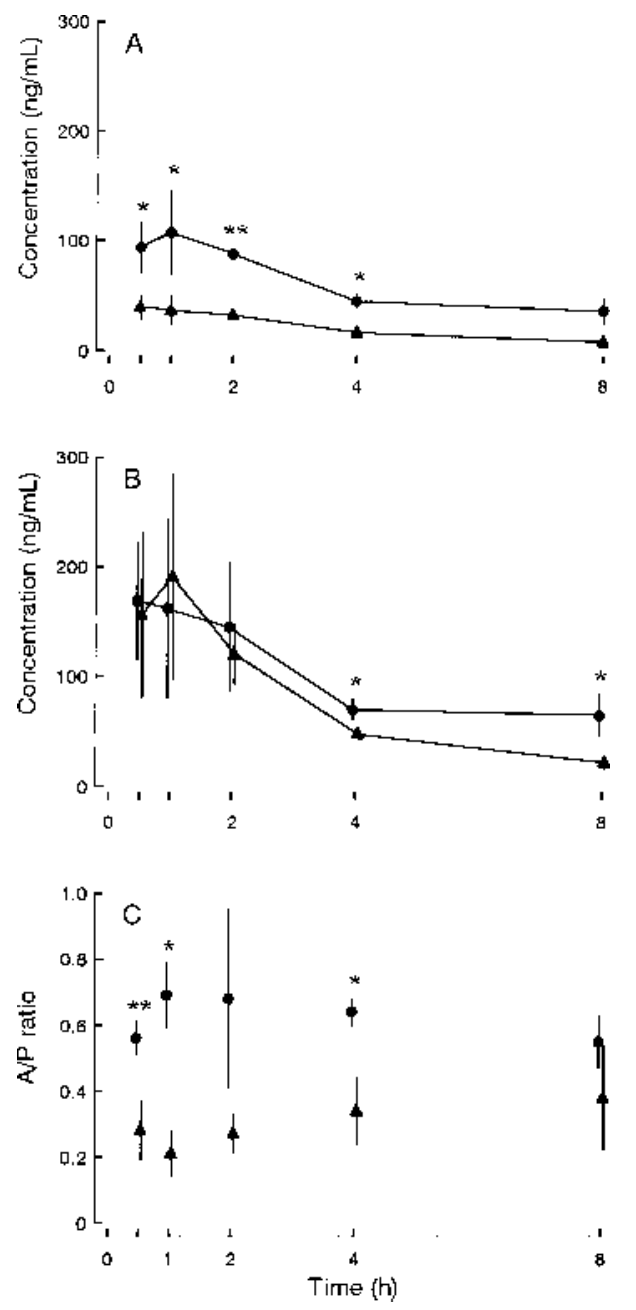

Fig. 2. Arterial and Portal Plasma Concentrations and the Ratios of Arterial to Portal Plasma Concentrations (A/P Ratios) of Both Enantiomers of BOF-4272 after the Oral Administration of Racemic BOF-4272 to Rats

Panels A, B, and C show the arterial plasma concentrations, the portal plasma concentrations, and the A/P ratios, respectively, of both enantiomers. The closed circles $(\boldsymbol{O})$ and triangles $(\boldsymbol{\Delta})$ show data for the $S(-)$ and $R(+)$ enantiomers, respectively. Each point represents mean \pm S.D. of 3 rats. The differences between the $S(-)$ and $R(+)$ enantiomers of BOF-4272 at the same time points were significant $(* p<0.05, * * p<0.01)$.

differences in portal plasma concentrations between the $S(-)$ and $R(+)$ enantiomers from $0.5 \mathrm{~h}$ to $2 \mathrm{~h}$ after oral administration (Fig. 2B). The A/P ratios of the $S(-)$ enantiomer were significantly higher than those of the $R(+)$ enantiomer from $0.5 \mathrm{~h}$ to $4 \mathrm{~h}$ after oral administration, and the $\mathrm{A} / \mathrm{P}$ ratios of both of the enantiomers remained relatively constant at all time points $(0.5,1,2,4,8 \mathrm{~h})$ after oral administration (Fig. 2C).

Table 1 shows the arterial, portal, and venous plasma concentrations and the difference between the portal and arterial plasma concentrations $(\Delta \mathrm{Cp})$ at $2 \mathrm{~h}$ after the oral administration of racemic BOF-4272 to rats and dogs. In both rats and dogs, the arterial and venous plasma concentrations of the enantiomers were almost the same, whereas the portal plasma concentrations of both enantiomers were higher than the arterial plasma concentrations. The arterial, portal, and venous plasma concentrations of the $S(-)$ enantiomer were higher than those of the $R(+)$ enantiomer in rats, whereas plasma concentrations of the $S(-)$ enantiomer were lower than those of the $R(+)$ enantiomer in dogs, after the oral ad- 
Table 1. Plasma Concentrations of BOF-4272 Enantiomers in Rats and Dogs

\begin{tabular}{lclll}
\hline \hline & $\begin{array}{c}\text { Arterial } \\
(\mathrm{ng} / \mathrm{ml})\end{array}$ & $\begin{array}{c}\text { Portal } \\
(\mathrm{ng} / \mathrm{ml})\end{array}$ & $\begin{array}{c}\text { Venous } \\
(\mathrm{ng} / \mathrm{ml})\end{array}$ & $\begin{array}{c}\Delta \mathrm{Cp} \\
(\mathrm{ng} / \mathrm{ml})\end{array}$ \\
\hline Rats & & & & \\
$S(-)$ enantiomer & $64 \pm 14$ & $145 \pm 47$ & $68 \pm 14$ & $81 \pm 36$ \\
$R(+)$ enantiomer & $38 \pm 9 * *$ & $113 \pm 37 * *$ & $39 \pm 9 * *$ & $75 \pm 32^{*}$ \\
Dogs & & & & \\
$S(-)$ enantiomer & $100 \pm 46$ & $293 \pm 186$ & $99 \pm 48$ & $193 \pm 153$ \\
$R(+)$ enantiomer & $144 \pm 75^{*}$ & $408 \pm 261 *$ & $139 \pm 76$ & $264 \pm 206$
\end{tabular}

Data in rats and dogs are shown as mean \pm S.D. of 10 and 5 animals, respectively. The differences in plasma concentration between the $S(-)$ and $R(+)$ enantiomers of BOF4272 were significant $(* p<0.05, * * p<0.001) . \Delta \mathrm{Cp}$ is the difference between portal and arterial plasma concentrations.

Table 2. Distribution to Liver of BOF-4272 Enantiomers in Rats and Dogs

\begin{tabular}{lcc}
\hline \hline & $\begin{array}{c}\text { Liver concentration } \\
(\mathrm{ng} / \mathrm{g} \text { tissue })\end{array}$ & $\mathrm{Kp}$ \\
\hline Rats & & \\
$S(-)$ enantiomer & $348 \pm 77$ & $5.5 \pm 1.2$ \\
$R(+)$ enantiomer & $798 \pm 194^{* *}$ & $21.3 \pm 4.4^{* *}$ \\
Dogs & $1741 \pm 894$ & $16.8 \pm 3.6$ \\
$S(-)$ enantiomer & $3414 \pm 1480^{*}$ & $26.0 \pm 9.7$ \\
$R(+)$ enantiomer & & \\
\hline
\end{tabular}

Data in rats and dogs are shown as mean \pm S.D. of 10 and 5 animals, respectively. The differences between the $S(-)$ and $R(+)$ enantiomers of BOF-4272 were significant $(* p<0.01, * * p<0.001) . \mathrm{Kp}$ is the ratio of liver concentration to arterial plasma concentration.

Table 3. Distribution to Kidney of BOF-4272 Enantiomers in Rats and Dogs

\begin{tabular}{lcl}
\hline \hline & $\begin{array}{c}\text { Kidney concentration } \\
(\mathrm{ng} / \mathrm{g} \text { tissue })\end{array}$ & $\mathrm{Kp}$ \\
\hline Rats & $395 \pm 123$ & $6.2 \pm 1.6$ \\
$S(-)$ enantiomer & $266 \pm 78^{* * *}$ & $7.0 \pm 1.3^{* *}$ \\
$R(+)$ enantiomer & $1066 \pm 495$ & $10.8 \pm 3.6$ \\
Dogs & $2308 \pm 841^{* *}$ & $17.9 \pm 5.6^{*}$ \\
$S(-)$ enantiomer & & \\
$R(+)$ enantiomer & &
\end{tabular}

Data in rats and dogs are shown as mean \pm S.D. of 10 and 5 animals, respectively. The differences between the $S(-)$ and $R(+)$ enantiomers of BOF-4272 were significant $(* p<0.05, * * p<0.01, * * * p<0.001) . \mathrm{Kp}$ is the ratio of kidney concentration to arterial plasma concentration.

ministration of racemic BOF-4272.

The concentrations and $\mathrm{Kp}$ values of the enantiomers in the liver are shown in Table 2. The liver concentration of the $S(-)$ enantiomer was significantly lower than that of the $R(+)$ enantiomer in both rats $(p<0.001)$ and dogs $(p<0.01)$; the $\mathrm{Kp}$ values in the liver of the $R(+)$ enantiomer were 3.87 times and 1.55 times higher than those of the $S(-)$ enantiomer, respectively. The concentrations and $\mathrm{Kp}$ values of the enantiomers in the kidney are shown in Table 3. The kidney concentration of the $S(-)$ enantiomer was significantly higher than that of the $R(+)$ enantiomer in rats $(p<0.001)$ and significantly lower than in dogs $(p<0.01)$. The Kp values in the kidney of the $R(+)$ enantiomer were 1.13 times and 1.66 times higher than those of the $S(-)$ enantiomer in rats and dogs, respectively.

The $S(-) / R(+)$ ratios of the enantiomers are shown in Table 4. In rats, the $S(-) / R(+)$ ratio for portal plasma was
Table 4. $S(-) / R(+)$ Ratios of BOF-4272 Enantiomers in Rats and Dogs

\begin{tabular}{lcc}
\hline \hline & Rats & Dogs \\
\hline Arterial plasma concentration & $1.72 \pm 0.23$ & $0.73 \pm 0.12$ \\
Venous plasma concentration & $1.75 \pm 0.20$ & $0.74 \pm 0.11$ \\
Portal plasma concentration & $1.30 \pm 0.08$ & $0.72 \pm 0.07$ \\
$\Delta \mathrm{Cp}$ & $1.07 \pm 0.11$ & $0.70 \pm 0.09$ \\
Liver concentration & $0.45 \pm 0.08$ & $0.49 \pm 0.09$ \\
Kp in the liver & $0.26 \pm 0.04$ & $0.69 \pm 0.22$ \\
Kidney concentration & $1.49 \pm 0.22$ & $0.45 \pm 0.10$ \\
Kp in the kidney & $0.87 \pm 0.12$ & $0.61 \pm 0.13$ \\
\hline
\end{tabular}

Data in rats and dogs are shown as mean \pm S.D. of 10 and 5 animals, respectively.

lower than that for arterial or venous plasma. In dogs, the $S(-) / R(+)$ ratios for arterial, venous, and portal plasma were all about 0.7 . The $S(-) / R(+)$ ratios for $\Delta \mathrm{Cp}$ were 1.07 and 0.70 in rats and dogs, respectively. The $S(-) / R(+)$ ratios for $\mathrm{Kp}$ in the liver were 0.26 and 0.69 , respectively, and the $S(-) / R(+)$ ratios for $\mathrm{Kp}$ in the kidney were 0.87 and 0.61 , respectively.

\section{DISCUSSION}

The present study has demonstrated the stereoselectivity of the plasma and liver concentrations of BOF-4272 enantiomers after oral administration to rats and dogs. Stereoselectivity is known to result from one or more pharmacokinetic processes. We estimated local intestinal absorption into the portal system and the hepatic first-pass effect using portal-venous concentration differences. Plasma samples are usually obtained near the maximum concentration, from $0.5 \mathrm{~h}$ to $4 \mathrm{~h}$ in rats ${ }^{12,13)}$ and from $1 \mathrm{~h}$ to $4 \mathrm{~h}$ in dogs. ${ }^{14)}$ In the present study, the $\mathrm{A} / \mathrm{P}$ ratios of both enantiomers remained relatively constant at all time points $(0.5,1,2,4,8 \mathrm{~h})$ after oral administration of racemic BOF-4272 to rats. Therefore, the concentrations of BOF-4272 enantiomers were measured at $2 \mathrm{~h}$ after oral administration to rats and dogs.

In rats, the $\Delta \mathrm{Cp} /$ portal concentration ratios of the $S(-)$ and $R(+)$ enantiomers were 0.56 and 0.66 , respectively. These values are the hepatic extraction ratios of the enantiomers normalized to the portal concentration. Furthermore, the $S(-) / R(+)$ ratio of these values in rats was 0.85 . The hepatic extraction of the $R(+)$ enantiomer from portal plasma was higher than that of the $S(-)$ enantiomer. Kp in the liver of the $R(+)$ enantiomer was 3.87 times higher than that of the $S(-)$ enantiomer. The $S(-) / R(+)$ ratio for portal plasma was lower than that for arterial or venous plasma. The hepatic extraction ratio of the $R(+)$ enantiomer of BOF-4272 was significantly greater than that of the $S(-)$ enantiomer in rats. ${ }^{11)}$ These results suggest that absorption from the intestinal tract into the portal system in rats was almost the same for the two enantiomers, whereas the hepatic uptake of the $R(+)$ enantiomer was greater than that of the $S(-)$ enantiomer.

In dogs, the $\Delta \mathrm{Cp} /$ portal concentration ratios of the $S(-)$ and $R(+)$ enantiomers were 0.66 and 0.65 , respectively, and the $S(-) / R(+)$ ratio of these values was 1.02 . This suggests equal hepatic extraction of the two enantiomers. The $\mathrm{Kp}$ in the liver of the $R(+)$ enantiomer was 1.55 times higher than that of the $S(-)$ enantiomer; however, the arterial and venous plasma concentrations of the $R(+)$ enantiomer were higher 
than those of the $S(-)$ enantiomer. The $S(-) / R(+)$ ratios were almost the same for arterial, venous, and portal plasma. These results suggest that absorption from the intestinal tract into the portal system of the $R(+)$ enantiomer was greater than that of the $S(-)$ enantiomer in dogs, whereas the hepatic uptake of the two enantiomers was almost the same. The finding that the $\mathrm{Kp}$ in the liver of the $R(+)$ enantiomer was higher than that of the $S(-)$ enantiomer may have been due to differences in the metabolism and/or elimination in bile of the two enantiomers in the dog liver.

The $S(-) / R(+)$ ratios for Kp in the kidney were 0.87 and 0.61 in rats and dogs, respectively. These results suggest that the renal uptake of the $R(+)$ enantiomer was greater than that of the $S(-)$ enantiomer in both animals. However, the stereoselectivity of the renal uptake of BOF-4272 enantiomers has little effect on the stereoselectivity of BOF-4272 in the systemic circulation in both animals. This may be because the cumulative urinary excretion of BOF-4272 after oral administration to both animals has been reported to be lower than $2 \%$. $^{13,14)}$

The stereoselectivity of the uptake of BOF-4272 by isolated rat hepatocytes, and thus in rats, is due to differences in the affinity of the enantiomers to the active transporter. ${ }^{15)}$ The difference in stereoselectivity in rats and dogs may be due to the presence of a variety of transporters. Therefore, we consider that it may be possible to predict the stereoselective pharmacokinetics of BOF-4272 in humans by performing experiments in isolated human hepatocytes.

In conclusion, the stereoselective pharmacokinetics of BOF-4272 in the systemic circulation is related to hepatic uptake in rats, but to absorption from the intestinal tract into the portal system in dogs.

\section{REFERENCES}

1) Sato S., Tatsumi K., Nishino T., Adv. Exp. Med. Biol., 309A, 135-138 (1991).

2) Uematsu, T., Nakashima, M., J. Pharmacol. Exp. Ther., 270, 453-459 (1994).

3) Yamamoto T., Moriwaki Y., Suda M., Nasako Y., Takahashi S., Hiroishi K., Nakano T., Hada T., Higashino K., Biochem. Pharmacol., 46, 2277-2284 (1993).

4) Iwahara R., Emoto S., Kaga J., Imura Y., Aoki M., Asakawa K., Iyakuhin Kenkyu, 25, 426-437 (1994). (in Japanese)

5) Matsugi M., Hashimoto K., Inai M., Fukuda N., Furuta T., Minamikawa J., Otsuka S., Tetrahedron: Asymmetry, 6, 2991-3000 (1995).

6) Okamoto K., Nishino T., J. Biol. Chem., 270, 7816-7821 (1995).

7) Suzuki H., Suematsu M., Ishii H., Kato S., Miki H., Mori M., Ishimura Y., Nishino T., Tsuchiya M., J. Clin. Invest., 93, 155-164 (1994).

8) Nishimura M., Yamaoka K., Yasui H., Naito S., Nakagawa T., Biol. Pharm. Bull., 17, 1301-1304 (1994).

9) Nishimura M., Yamaoka K., Yasui H., Naito S., Nakagawa T., Biol. Pharm. Bull., 18, 980-983 (1995).

10) Nishimura M., Yamaoka K., Naito S., Nakagawa T., Biol. Pharm. Bull., 19, 1197-1202 (1996).

11) Nishimura M., Yamaoka K., Naito S., Nakagawa T., Biol. Pharm. Bull., 20, 1285-1289 (1997).

12) Naito S., Nishimura M., Nogawa H., J. Pharm. Pharmacol., 51, 347 351 (1999).

13) Naito S., Nishimura M., Pharm. Pharmacol. Commun., 5, 123-131 (1999).

14) Naito S., Nishimura M., Yoshitsugu H., Nogawa H., Biol. Pharm. Bull., 22, 1391-1395 (1999).

15) Naito S., Nishimura M., Yakugaku Zasshi, 121, 989 -994 (2001). 